\title{
BIOMARKER EXPRESSION IN BREAST CANCER. CORRELATIONS WITH OUTCOME FOLLOWING NEOADJUVANT CHEMOTHERAPY
}

Augusto Ribeiro Gabriel', Ruffo Freitas-Junior ${ }^{1}$

${ }^{1}$ CORA - Centro Avançado de Diagnóstico da Mama, Universidade Federal de Goiás, Rede Brasileira de Pesquisa em Mastologia - Goiânia (GO), Brazil.

Purpose: Breast cancer is the most common type of cancer in women in Brazil and worldwide, corresponding to around $20 \%$ of all malignant tumors in women. Neoadjuvant chemotherapy improves survival rates in around $30 \%$ of patients with locally advanced disease. This study evaluated biomarker expression in tumor samples and assessed outcome variables following neoadjuvant chemotherapy. Methods: This longitudinal, analytical study included 86 patients of 28 to 80 years of age who were evaluated according to clinical data, histology and laboratory analysis of selected markers. Results: Positivity for estrogen (ER) and progesterone receptors (PR) was $58.54 \%$ and $65.79 \%$, respectively. The tumors were predominantly negative for the HER2, CK5/6, EGFR, p53 and Topo II biomarkers and predominantly positive for the Ki-67, PTEN and IGF-1 biomarkers. The results obtained for ER showed associations with PR ( $\mathrm{p}=0.002), \mathrm{Ki}-67$ ( $\mathrm{p}=0.049)$, CK5/6 ( $\mathrm{p}=0.027)$ and p53 ( $\mathrm{p}=0.032)$. Likewise, there were associations between HER2 and Ki-67 ( $\mathrm{p}=0.028)$ and between Ki-67 and Topo II ( $\mathrm{p}=0.001)$. Eighteen patients $(20.93 \%)$ achieved complete clinical response, while $9(10.47 \%)$ achieved pathologic complete response (pCR). There was an association between pCR and the HER2 ( $p=0.05$ ) and Topo II ( $p=0.007)$ biomarkers. More patients with HER2 tumors (40\%) achieved pCR compared to those with the other subtypes, while none of the patients with luminal A tumors achieved pCR ( $\mathrm{p}=0.043)$. Conclusions: In this study, HER2 and Topo II expression, as well as tumors classified as HER2 subtype or luminal A subtype were predictive of pCR. 